\title{
The Extraction of Depth Discontinuities Using Disparity Map for Human Visual Fatigue
}

\author{
Gil Ja So, Sang Hyun Kim, and Jun Yeop Kim, Member, IACSIT
}

\begin{abstract}
The complexity of the world is a factor of evaluating the visual fatigue of $3 \mathrm{D}$ video. Depth discontinuities are the layers of the world and can be a characteristic of the complexity of the world. We suppose a new method to extract a depth discontinuities from a disparity map. We employed DIP algorithm for extraction of the depth discontinuities with a learned threshold automatically inferred based on the average and standard deviation of the disparity, that is, depth of the stereoscopy.
\end{abstract}

Index Terms-Depth discontinuities, DIP, disparity map, human visual fatigue.

\section{INTRODUCTION}

Our eyes have its own viewpoint of the world and both eyes receive slightly different images, left and right image. Stereoscopy is the perception of the depth of $3 \mathrm{D}$ with the disparity of the two image. The reason which users feel fatigue as seeing stereoscopic movies is that stereoscopic image is created as the way that the human unify the differences between the two eyes to form the realistic illusion. These two images then shown to the viewer by way of binocular arrangement.

The technology to present stereoscopic images to viewer have to consider the difference of the binocular disparity of the viewers and the ability of the unifying the two images in the brain.

Contents such as movies, animations, games can be produced with the stereoscopic technology. However stereoscopic images are hard to get comfortable images. Many various directing techniques are used to increase the three-dimensional illusion for viewers [1], [2]. However, Testing after production of contents is complete is the most expensive. We need to develop a evaluation method to test fatigue of viewer when sees stereoscopic images.

Factors causing fatigue of viewer in stereoscopic contents can be divided into two, spatial factor and temporal factor. Temporal factors are horizontal or vertical movements, the velocity of movement, scene transitions, et al. [3]-[7], spatial factors are the complexity of objects, the amount of the number of objects which would make up the background in contents, and the nature of the texture of objects, color or brightness.

Manuscript received November 10, 2013; revised January 15, 2014.

G. J. So is with the Department of Cyber \& Police Science of the University of Young-San at Yangsan, Kyungnam (e-mail: kjso@ ysu.ac.kr).

S. H. Kim is with the Department of Computer Engineering of the University of Young-San at Yangsan, Kyungnam (e-mail: ksh50@ ysu.ac.kr).

J. Y. Kim is with the School of Undeclared Majors, University Collage of the University of Young-San at Busan (e-mail: neocopy@ ysu.ac.kr).
Depth discontinuities help a user to distinguish an object from background or others and get a knowledge on layers of the world . Depth discontinuities is defined as a point on the image plane where the depth field is discontinuous [8]. Depth discontinuity is a powerful clue for many real-tasks like as tracking, detection, image retrieval. We use depth discontinuity for the evaluation of the human visual fatigue in 3D.

The complexity of the world which is a factor of the fatigue, that means the amount of the number of objects which would make up the background in the world, is represented with the distribution of the depth discontinuities.

Depth discontinuities are similar to edges in a $2 \mathrm{D}$ image. As an edge in a 2D image occur on the boundary between two different regions which have homogeneity and connectivity in an image, Depth discontinuity occurs on the boundary between different depth in an 3D image. A region is called homogeneous if all its pixels are homogeneous. A region is called connected if there exists a connected path between any two pixels.

Little and Gillett [9] used an average of the local region and matched a left and right image of stereoscopy. Afterwards matching, they inferred depth discontinuities from pixels of occluded regions. Toh and Forrest[10] defined a depth discontinuity as a boundary which a left and right do not match.

However most of these research used information on texture in the image, and can not extract well depth discontinuity on the boundary of the occluded region. To solve this problem Stan Birchfield and And Carlo [11] proposed pixel to pixel based depth discontinuity extraction algorithm.

This algorithm used an dynamic programming for the faster running time. However, this method does not enforce the inter-scanline inconsistency, leads to the horizontal "streaking" artifacts [12], [13]. To reduce this problem, [11] propagates information is need between scanlines in post production.

We adopt DIP algorithm to get reliable depth discontinuity. DIP is the algorithm for edge detection using a human visual system which is more sensitive to the edges and valleys in dark regions than those in bright regions [14], [15]. We extract depth discontinuity from depth map by DIP. DIP needs a appropriate threshold to decide whether is discontinuous. This needs a user's interaction during process. We infer a threshold by the characteristics of the distribution of the depth map, that is, mean and standard deviation.

The paper is organized as follows. We would briefly review related previous works and describe our proposed method in Section III. We show the effect of the threshold in DIP and the evaluation formula to get appropriate threshold for extracting 
depth discontinuity from disparity map by DIP in Section IV. In Section V contains some of our conclusion.

\section{RELATED WORKS}

\section{A. Pixel to Pixel Depth Discontinuities}

An algorithm which extract depth discontinuities based on Pixel to Pixel (throughout this paper, this algorithm would be called P2P) label pixels which are greater than threshold, and extract these pixels as depth discontinuity. Advantage of this method is that depth discontinuity can be extracted without texture, is robust in image sampling, process time is fast by dynamic programming. Postprocessor of this method propagates disparity map vertically to get clearer disparity map.

$\mathrm{P} 2 \mathrm{P}$ produces the disparity map using left and right image of stereo images using intensity based stereo matching algorithms. P2P match pixels one scanlines of the left or right image to pixels in the corresponding pixels of other. These matched pixels are arrayed in the form of match sequences. Fig. 1 shows a match sequence on an short scanline. Unmatched pixels are occluded. depth discontinuity pixels occur at the right of occluded pixels in the left image and at the left of occluded pixels in the right image in stereoscopy.

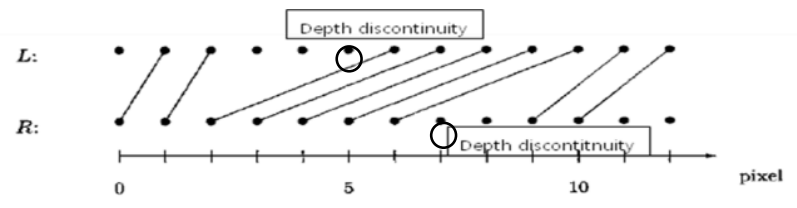

Fig. 1. The example of match sequence,

$M=\langle(1,0),(2,1),(6,2),(7,3),(8,4),(9,5),(10,6),(11,9),(12,10),(13.11)\rangle$ The depth discontinuity pixels are circled.

$\mathrm{P} 2 \mathrm{P}$ propose a simple cost function to select a best sequence as shown in (1). The best match sequence has the lowest cost.

$$
\gamma(m)=N_{o c c} K_{o c c}-N_{m} K_{r}+\sum_{i=1}^{N_{m}} d\left(x_{i}, y_{i}\right)
$$

In (1), $K_{o c c}$ is the occlusion penalty constant, $K_{r}$ is the match reward constant, $d\left(x_{i}, y_{i}\right)$ is the distance between pixel $x_{i}$ and pixel $y_{i}$, which is the pixel of the left and right stereo image, each. $N_{o c c}$ is the number of occlusion. $N_{m}$ is the number of matched pairs.

$\mathrm{P} 2 \mathrm{P}$ searches for the best possible path to make a cost of the sequence the lowest by the technique of dynamic programming. The process of finding a best match sequence in one scanline is independent of other scanlines. However, the intensity values of pixels from different scanlines are not independent. Thus $\mathrm{P} 2 \mathrm{P}$ needs a post process which propagate information from rows and columns together to use all the information in the images.

After post process, depth discontinuity is selected as a point which are accompanied by changes of at least two disparity levels.

\section{B. $D I P$}

DIP is the algorithm to detect edges which are the regions involving abrupt changes of intensity, and valleys which are the regions composed of local intensity minima.

Valley is very important in vision. Various methods to detect valley like as Laplacian, Pearson's logical valley use the gradient values of the pixels in a local region. However, Laplacian is too sensitive to noise and Pearson's logical valley cannot sometimes extract valleys which have somewhat small rates of change of intensity.

The entropy operator computes the entropy of intensity in a local region. This method depends on the local intensities, therefore, can extract the edges of dark regions very well. The disadvantage of this method is that it extracts edges as thick lines and cannot respond in valleys very well.

The human viewer is more sensitive to the edges and valleys in dark regions than those in bright regions. Therefore, to perceive and analyze objects in a manner akin to the human visual system, one must extract sketch features subject to the local intensities.

DIP satisfies the necessity for the perception and analysis of the human visual system as mentioned above. DIP is the in defined as (3).

$$
\begin{array}{r}
D P(m, n)=\frac{I_{m}(m, n)}{\bar{I}(m, n)}-\frac{I(m, n)}{\bar{I}(m, n)} \\
D I P(m, n)=D P \frac{\bar{I}(m, n) \bar{I}(m, n)}{I_{m}(m, n) I(m, n)}
\end{array}
$$

$I(m, n)$ is the intensity of the pixel $(m, n), \bar{I}_{m}(m, n)$ is the sum of intensities and $I_{m}(m, n)$ is the maximum intensity in a window, In (2) and (3). Due to $I_{m}(m, n)$, the value of DP depends on local intensities and difference make DP respond well in valleys and edges. However, DP has a defect of extracting neighbor pixels as well as the valleys themselves.

To remove this problem, if the difference of $I_{m}(m, n)$ and $I(m, n)$ is greater than a given threshold obtained in (5) is applied. Each $\bar{I}_{m}(m, n) \quad I_{m}(m, n)$ are much the same in valleys and neighbors, while $I(m, n)$ is smaller in the valleys than in their neighbors. Thus, the values of DIP are so much larger in the valleys themselves that it extracts valleys thinly.

\section{PROPOSED METHOD}

The structure of the proposed system is consist of four step, disparity map calculation, noise reduction, post processing, discontinuities extraction.

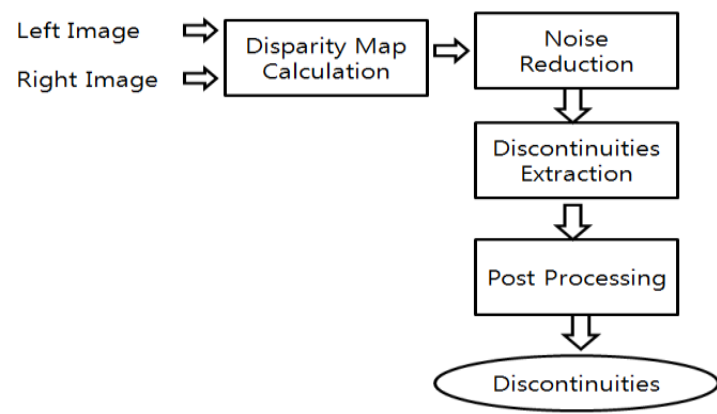

Fig. 2. The structure of the proposed system. 
The proposed system is presented in Fig. 2. Disparity map is extracted from two images of the stereoscopy. Holes which occur in occluded regions should be filled before next process. Hole is filled with the interpolation of neighbor pixels. Next, Edge is detected from the pre-processed disparity map by DIP Detected edges from the disparity map are depth discontinuities. However, edges which occur in the horizontal lines of the desk, ground are thick. So, in post processing, edges are made to be thin.

\section{A. Disparity Map Calculation}

Disparity map is extracted by the method proposed by Andres Geiger[16]. This method decrease stereo matching ambiguities using a prior distribution estimated from robust support points. Support points are reliable correspondences, which give valuable information to disambiguate remaining ambiguous disparities. 2D mesh via Delaunay triangulation is computed using a sparse set of support points. When creating $2 \mathrm{D}$ mesh, a prior is used to make the process efficient by restricting the search to plausible regions.

\section{B. Noise Reduction}

There are many holes in the disparity map due to occluded region. In the next step, edge detection from the disparity map by DIP, pixels would be the denominator of the equation to calculate the gradient of the depth. If the value of the pixel is a zero, this pixel is neglected in the extraction of the edge, that is, depth discontinuity because of the reason above mentioned reason. However, neglecting the zero pixel, the hole, would be the cause of the disconnected edge in edge detection by DIP. Therefore, the hole should be filled with non-zero value.

To fill these holes, we calculate the value of pixel in holes using the horizontally and vertically interpolating value of the pixel in the holes' boundary. Equation (3) shows the interpolation of the pixel in the hole with the nearest neighbor pixels.

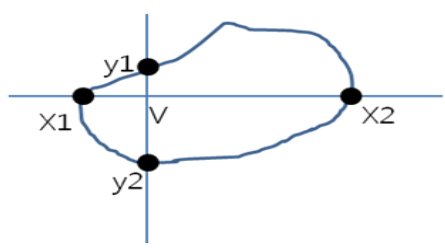

Fig. 3. Pixel v in occluded region.

The pixel $v$ is a pixel in the hole, $x_{1}, x_{2}, y_{1}, y_{2}$ are pixels in the boundary which meets horizontally and vertically extended line. If $v$ is in the occluded region, the intensity of the $v$ is interpolated with the differently weighted $x_{1}, x_{2}, y_{1}, y_{2}$.

$$
\begin{gathered}
I(v)=\frac{\left(x_{1} w_{1}+x_{2} w_{2}\right)+\left(y_{1} w_{3}+y_{2} w_{4}\right)}{2} \\
w_{1}=\frac{\operatorname{dist}\left(x_{1}, v\right)}{\operatorname{dist}\left(x_{1}, x_{2}\right)} \quad w_{2}=\frac{\operatorname{dist}\left(x_{2}, v\right)}{\operatorname{dist}\left(x_{1}, x_{2}\right)} \\
w_{3}=\frac{\operatorname{dist}\left(y_{1}, v\right)}{\operatorname{dist}\left(y_{1}, y_{2}\right)} w_{4}=\frac{\operatorname{dist}\left(y_{2}, v\right)}{\operatorname{dist}\left(y_{1}, y_{2}\right)}
\end{gathered}
$$

$\operatorname{dis}(x, y)$ is the distance of pixel $x$ and $y$.

In (4), $w_{1}, w_{2}, w_{3}, w_{4}$ is obtained with the ratio of the distance from pixel $v$ to neighbor pixels.
We present the algorithm to interpolate all pixels of the occluded region.

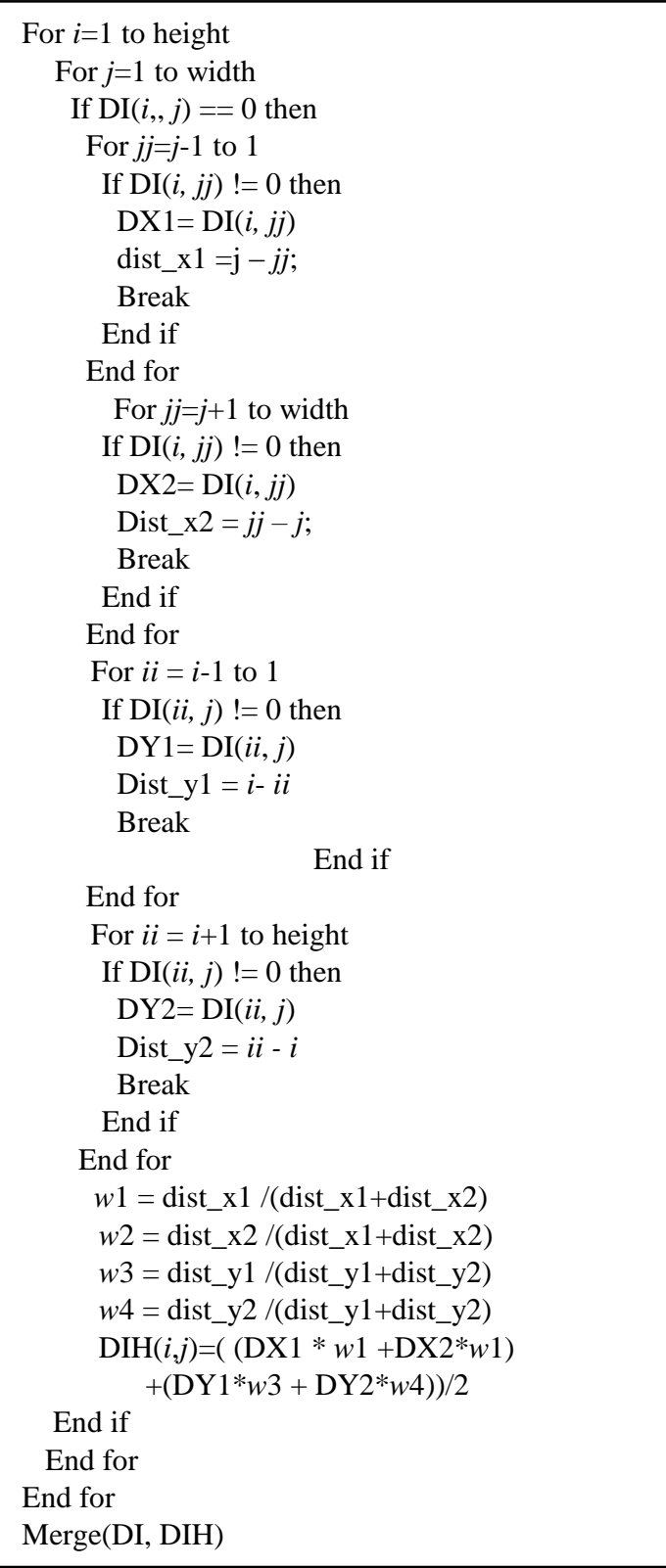

Fig. 4. Interpolation algorithm of the intensity of the pixel in the occluded region.

\section{The Extraction of the Depth Discontinuities}

DIP is the algorithm to extract edges from 2D image using the information of the intensity of pixels. The intensity of the $2 \mathrm{D}$ image is the brightness or materials in contrast with the depth or layers of the disparity map. Because of the similarity of the depth discontinuity in depth map and the edge of the image in the side of the using of the information of the gradation of the intensity, DIP could extract depth discontinuities from disparity map. DIP extract a pixel as an edge when the difference is lower than a threshold.

The result of the extraction is very various as to a threshold. As a threshold is so high, edge would be extracted too much. On the contrary to this, as a threshold is too low, edge would be extracted too less. Therefore, threshold is very important in the extraction of depth discontinuities. Usually, threshold is decided by user during the process. The interaction of the user during the process is improper to use depth discontinuity as a 
factor of the evaluation of the visual fatigue. We need a way to extract depth discontinuities to evaluate the visual fatigue in real time. To extract depth discontinuities in real time, a threshold should be decided without the interaction of the user efficiently.

Because the threshold should be decided to represent the characteristics of the disparity map, we learned the threshold with some disparity map and made a evaluation formula in (5) to decide a threshold to be representative the disparity map.

The difference of the intensity of the disparity map means the depth of the difference, so, the value of threshold could be measured with mean and standard deviation of the depth.

$\operatorname{Threshold}(x)=-0.029 \times M(x)+0.014 \times S(x)+4.884$

In (5), $M(x)$ is the mean and $S(x)$ is the standard deviation of the image $x$.

\section{Post Process}

The final process is a thinning after edge detected. If Object has a vertically different depth, there can be a thick edge during discontinuity extraction, so we have to thin a thick edge after edge detection. We employed zhang and seun thinning algorithm[16]. This thinning method obtain a new value from the previous iteration value. This method is called parallel method. This method is fast and simple to be implemented. So we would make a thick edge to be thin in depth Discontinuities.

\section{EXPERIMENT}

\section{A. The Comparison of Depth Discontinuity of P2P and DIP}

We extracted a depth discontinuity by P2P and DIP. (c) and (d) in Fig. 5 shows the result of P2P and DIP each.

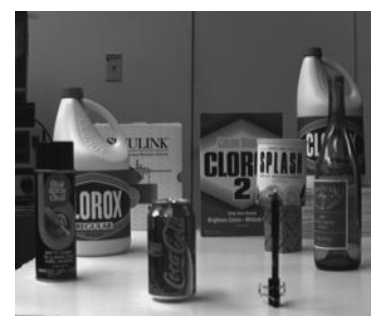

(a) source of image

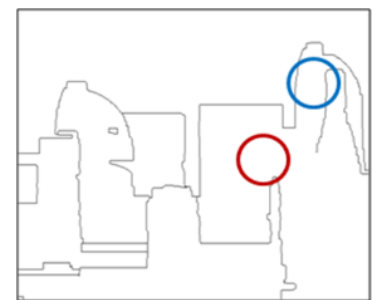

(c) depth discontinuity by $\mathrm{P} 2 \mathrm{P}$

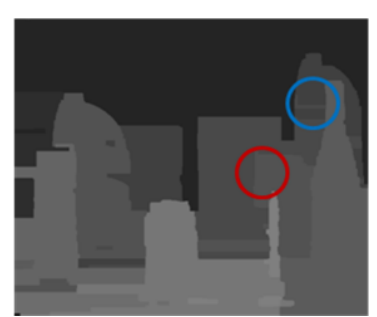

(b) disparity map

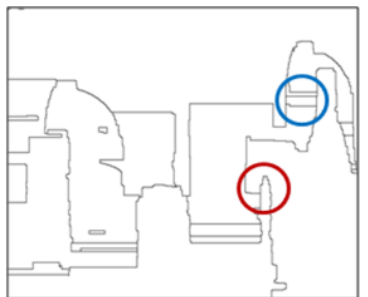

(d) depth discontinuity by DIP
Fig. 5. The result of the depth discontinuity extracted by $\mathrm{P} 2 \mathrm{P}$ and DIP.

In Fig. 5, P2P cannot find the horizontal edge between box and can, in contrary, DIP can find it. The result of the disparity map is clearer, depth discontinuities are the more accurate. Therefore, we use 3D depth map made by Middlebury to learn the threshold of the DIP.

\section{B. Learning the Threshold of DIP}

We present experimental results to show possibility for automatically learning the threshold used in DIP algorithm with closeness to the result of hand-tuned threshold. To obtain the more accurate learning data, we used disparity set in the Middlebury dataset.

\section{1) Environment of the experiment}

Fig. 6 shows the left image set from the Middlebury dataset [18]. Fig. 7 shows the disparity map from the Middlebury dataset corresponding to the image of Fig. 6.

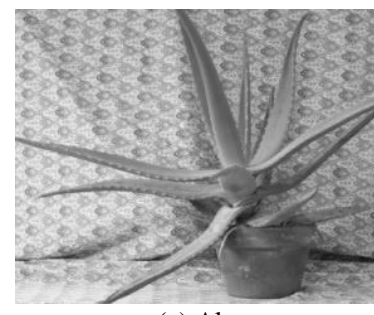

(a) Aloe

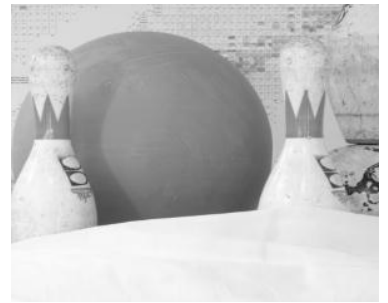

(c) Bowling

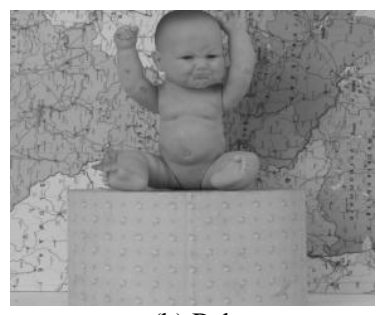

(b) Baby

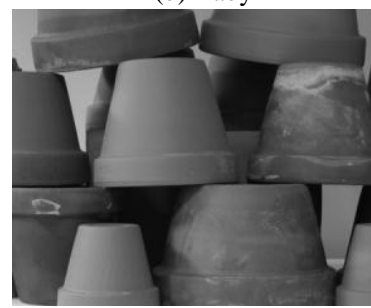

(d) Flowerspot
Fig. 6. Left image of stereoscopy in Middlbury 3D database.

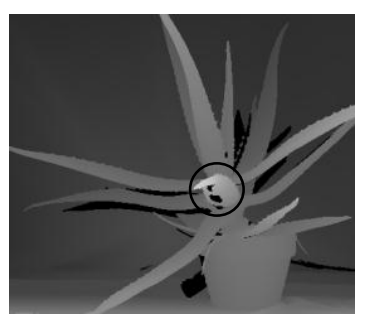

(a) Disparity map of Aloe

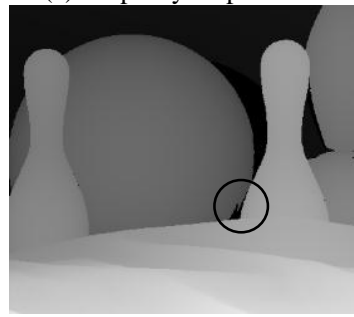

(c) Disparity map of Bowling Fig. 7. Disparity n

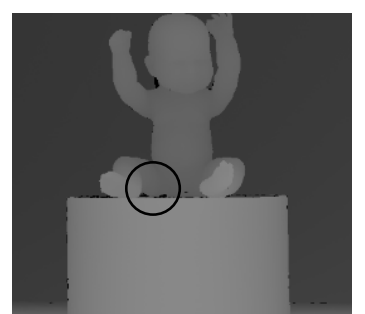

(b) Disparity map of Baby

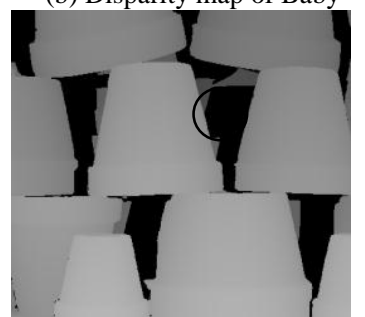

(d) Disparity map of Flowerspot opy in Middlbury 3D database.
Disparity map in Fig. 7 is obtained by using technique of [18] and published in [19], [20]. All of images have some occluded regions that are marked with circle. We filled this hole with the interpolation method that is presented in (3) before learning process.

\section{2) The result of depth discontinuities using DIP}

We extracted depth discontinuities from Aloe showed in Fig. 8. Because of the threshold is various, the results are different as to the used threshold. Fig. 8 shows that there is the correlation between the threshold and the depth discontinuities extracted by DIP.

In Fig. 8, the best result is the (e) which is extracted with 
threshold 5. Depth discontinuities is less than expected from (a) to (d), more in (f). As Fig. 8 shows, the result of the extraction is very different as to the value of the threshold. We need a way to learn the threshold automatically in real time to evaluate the visual fatigue.

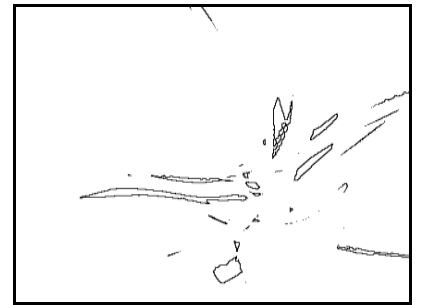

(a) Threshold $=1$

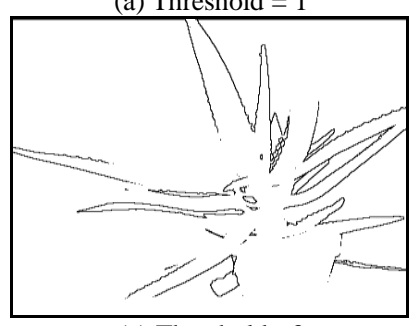

(c) Threshold $=3$

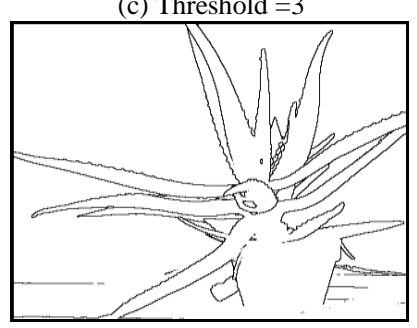

(e) Threshold $=5$

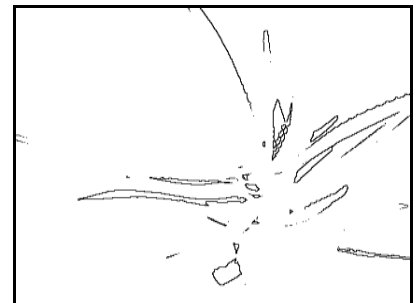

(b) Threshold $=2$

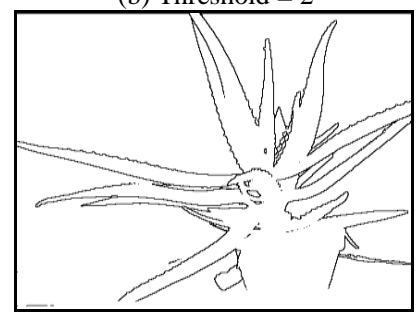

(d) Threshold=4

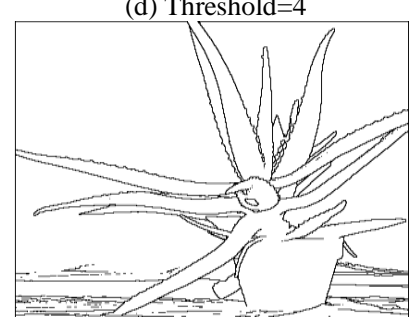

(f) Threshold $=6$
Fig. 8. The result of depth discontinuities by DIP as to various thresholds.

\section{3) Learning the threshold}

We learned threshold with the 10 image set in Middlebury dataset. We infer a threshold by the characteristics of the distribution of the depth map, that is, average and standard deviation. Table I shows average, standard deviation of the 10 image each and the result of the learned threshold.

\begin{tabular}{|c|c|c|c|c|}
\hline Name & $\begin{array}{c}\text { Average } \\
\text { (avg) }\end{array}$ & $\begin{array}{c}\text { Standard } \\
\text { Deviation } \\
\text { (std) }\end{array}$ & $\begin{array}{c}\text { Hand- } \\
\text { tuned } \\
\text { threshold }\end{array}$ & $\begin{array}{l}\text { Learned } \\
\text { threshold }\end{array}$ \\
\hline aloe & 70.18 & 30.07 & 5.00 & 3.26 \\
\hline baby & 83.03 & 32.18 & 2.00 & 2.92 \\
\hline bowling & 115.53 & 59.46 & 2.00 & 2.35 \\
\hline flowerpots & 124.35 & 54.34 & 3.00 & 2.03 \\
\hline lampshade & 104.13 & 50.37 & 3.00 & 2.56 \\
\hline midd & 93.98 & 48.95 & 3.00 & 2.83 \\
\hline monopoly & 73.50 & 42.94 & 2.00 & 3.34 \\
\hline plastic & 135.33 & 40.35 & 1.00 & 1.52 \\
\hline rocks & 107.44 & 35.98 & 2.00 & 2.27 \\
\hline wood & 121.33 & 39.38 & 2.00 & 1.91 \\
\hline
\end{tabular}

After learning with average and standard deviation, we get an evaluation to decide the threshold, following (6).

$$
\text { Learned threshold }=-0.029 \times \operatorname{avg}+0.014 \times \operatorname{std}+4.884
$$

Fig. 9 shows depth discontinuities by DIP used learned threshold for Aloe, Baby, Bowling, Flowerspot. Learned threshold would be truncated or rounded up to convert a floating number to a decimal.

Depth discontinuities of Fig. 9(a) are not sufficient compared with the hand-tuned result. From Fig. 9(b) to (d) is similar to the result of the hand-tuned threshold.

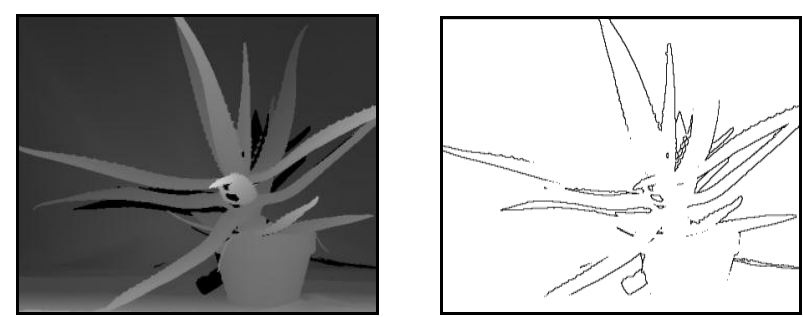

(a) depth discontinuites of Aloe with learned threshold 3

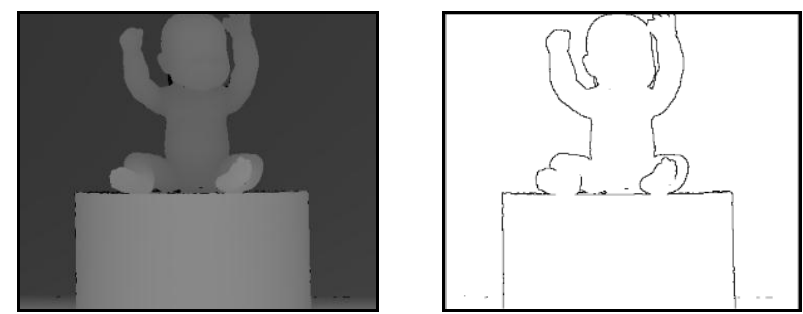

(b) depth discontinuites of Baby with learned threshold 3
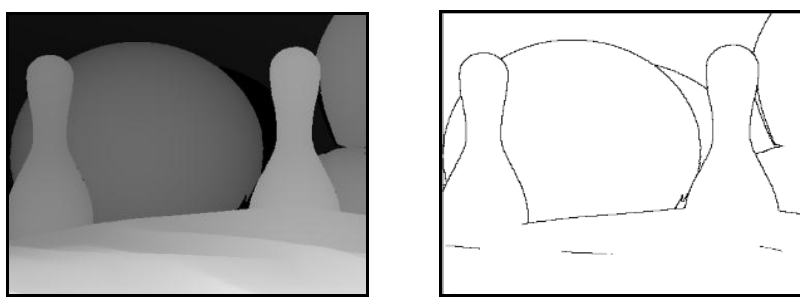

(c) depth discontinuites of bowling with learned threshold 2
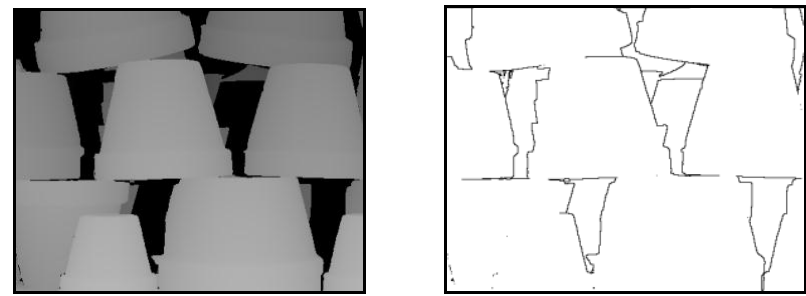

(d) depth discontinuites of Flowerspots with learned threshold 2 Fig. 9. The result of depth discontinuities by DIP with learned threshold.

\section{CONCLUSION}

Depth discontinuities are important factor to let a human to make layers of the world and to evaluate the visual fatigue of stereoscopic image/video. It is known that visual fatigue is caused by spatial and temporal complexity, spatial complexity of the world can be interpreted with depth discontinuities. Extracting depth discontinuities from disparity map is similar to extraction of edges in 2D image.

We employed DIP algorithm to extract depth discontinuities. However, DIP need a user interaction to decide a threshold. To apply to evaluation of the visual fatigue in real time, there needs to obtain a threshold in real time based on the disparity map. We learned threshold with the Middlebury image sets and made evaluation to decide a threshold based on the average and standard deviation of the disparity map. Afterwards, we will evaluate the visual fatigue with depth discontinuities.

\section{ACKNOWLEDGMENT}

This research is supported by Korea Creative Content Agency (KOCCA) in the Culture Technology (CT) Joint Research Center Project 2013. 


\section{REFERENCES}

[1] N. G. Kim, "Human factors for 3D stereoscopic cinema," Communications of KIISE, vol. 29, no. 8, pp. 48-54, Nov. 2011.

[2] S. H. Kim, M. J. Kim, and J. W. Kang, "An analysis of the visual characteristic of directing for three dimensional films," Journal of the Korea Contents Association, vol. 11, no. 2, pp. 229-237, 2011.

[3] Y. Nojiro, H. Yamanoue, A. Hanazato, M. Emoto, and F. Okano, "Visual comfort/discomfort and visual fatigue caused by stereoscopic HDTV viewing," in Proc. SPIE-IS\&T Electronic Imaging, vol. 5291, 2004.

[4] S. Yano, M. Emoto, and T. Mitsuhashi, "Two factors in visual fatigue caused by stereoscopic HDTV images," Displays 25, pp. 141-150, 2004.

[5] S. Yano, S. Ide, and T. Mitsuhashi, "A study of visual fatigue and visual comfort for 3D HDTV/HDTV images," Displays 23, pp. 191-201, 2002.

[6] M. Emoto, Y. Nojiri, and F. Okano, "Change in fusional vergence limit and its hysteresis after viewing stereoscopic TV," Displays 25, pp. 67-76, 2004.

[7] D. Kim, D. Min, J. Oh, S. Jeon, and K. Sohn, "Depth image quality metric for three-dimensional video," in Proc. SPIE-IS\&T, Electronic Imaging, 2009,pp. 723719_1-723719_2.

[8] S. Birchfield, "Depth and motion discontinuities," Ph.D. dissertation, Stanford Univ., June 1999.

[9] J. J. Little and W. E. Gillett, "Direct evidence for occlusion in stereo and motion," Image and Vision Computing, vol. 8, no. 4, pp. 328-340, Nov. 1990.

[10] P. S. Toh and A. K. Forrest, "Occlusion detection in early vision," in Proc. the 3rd International Conference on Computer Vision, 1990. pp. 126-132.

[11] S. Birchfield and C. Tomasi, "Depth discontinuities by pixel-to-pixel stereo," International Journal of Computer Vision, vol. 35, no. 3, pp. 269-293, 1999

[12] S. Chen, B. Mulgrew, and P. M. Grant, "A clustering technique for digital communications channel equalization using radial basis function networks," IEEE Trans. on Neural Networks, vol. 4, pp. 570-578, July 1993.

[13] L. Wang, M. Gong, R. Yang, and D. Nister, " High-Quality real-time stereo using adaptive cost aggregation and dynamic programming," in Proc. 3D Data Processing, Visualization, and Transmission, Third International Symposium, pp. 798-805, June 2006.

[14] Y. J. Ryoo and N. C. Kim, "Valley operator extracting sketch features:DIP," Electron. Lett., vol. 248, pp. 461-463, Apr. 1988.

[15] Y. D. Chun, S. Y. Seo, and N. C. Kim, "Image retrieval using BDIP and BVLC moments," IEEE Transactions on Circuits and Systems for Video Technology, vol. 13, no. 9, Sept. 2003.

[16] A. Geiger, M. Roser, and R. Urtasun, "Efficient large-scale stereo matching," in Proc. Asian Conference on Computer Vision, vol. 6492, Nov. 2010, pp. 25-38.

[17] T. Y. Zhang and C. Y. Suen, "A fast parallel algorithm for thinning digital patterns," Comm ACM, vol. 27, pp. 236-239, 1984

[18] D. Scharstein and R. Szeliski. "High-Accuracy stereo depth maps using structured light," in Proc. IEEE Computer Society Conference on Computer Vision and Pattern Recognition (CVPR 2003), vol. 1, pp. 195-202, June 2003.

[19] D. Scharstein and C. Pal, "Learning conditional random fields for stereo," in Proc. IEEE Computer Society Conference on Computer Vision and Pattern Recognition (CVPR 2007), Minneapolis, MN, June 2007.
[20] H. Hirschmüller and D. Scharstein, "Evaluation of cost functions for stereo matching," in Proc. IEEE Computer Society Conference on Computer Vision and Pattern Recognition (CVPR 2007), Minneapolis, MN, June 2007.

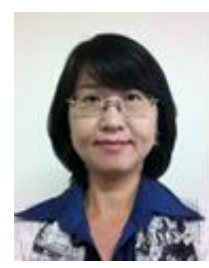

Gil Ja So received the diploma in computer science from the Dong-Eui University of Pusan, South Korea in 1994, MS and PhD degrees, both in natural language processing of computer engineering, from the Pusan National university, South Korea in 1997 and 2012, respectively. From September 2001, she is at the Department of Cyber \& Police Science of the University of Young-San at Yangsan, Kyungnam, as a lecturer. Her doctoral dissertation work focused on the Korean grammar checker for parsing Korean documents and correcting context-sensitive words in documents. The topics she has worked on include virtual reality, game development and artificial intelligence of non-players of game, and 3D stereoscopic images. Her current research is on region-based intensity correction in 3D stereoscopic images. She is a member of the KMMS (Korea Multimedia Society) and KIPS (Korea Information Processing Society).

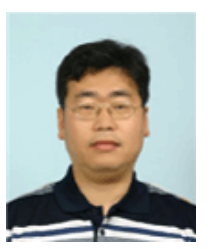

Sang Hyun Kim received the diploma in electronics engineering from the Kyung-Pook National University of Daegu, South Korea in 1991, MS and PhD degrees both in image processing of electronics engineering, from the same university, in 1993 and 1998, respectively. He was with the Samsung Medison Co. Ltd, in Korea as a senior researcher from 1998 to 2001. From September 2001, he is at the Department of Computer Engineering of the University of Young-San at Yangsan, Kyungnam, as an associate professor His doctoral dissertation work focused on the development of fractal image coding method and transmission for image processing, image communication and computer vision problems. The topics he has worked on include virtual reality, ultrasound image processing, medical image processing, image feature extraction and classification, and 3D stereoscopic images. His current research is on region-based intensity correction in 3D stereoscopic images. He is a member of the KMMS (Korea Multimedia Society) and KIPS (Korea Information Processing Society).

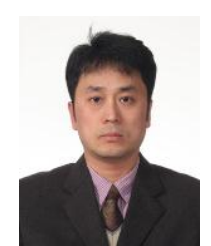

Jeong Yeop Kim received the diploma in electronics engineering from Kyung-Pook National University of Daegu, South Korea in 1990, MS and PhD degrees both in image processing of electronics engineering, from the same university, in 1992 and 2000, respectively. He was with the Samsung Advanced Institute of Technology in Korea as a senior research staff from 1994 to 2000. From March 2001, he is at the School of Undeclared Majors, University Collage of the University of Young-San at Busan, as an associate professor. His doctoral dissertation work focused on detecting and adjusting the white point from single color image. The topics he has worked on include color printing, error diffusion, color correction and 3D stereoscopic images. His current research is on correction of color data in 3D stereoscopic images. He is a member of the KMMS (Korea Multimedia Society) and KIPS (Korea Information Processing Society). 